\title{
Observation of Laser Power Amplification in a Self-Injecting Laser Wakefield Accelerator
}

\author{
M. J. V. Streeter, ${ }^{1,2,3}$ S. Kneip, ${ }^{3}$ M. S. Bloom, ${ }^{3}$ R. A. Bendoyro, ${ }^{4}$ O. Chekhlov, ${ }^{5}$ A. E. Dangor, ${ }^{3}$ A. Döpp, ${ }^{3,6,7}$ \\ C. J. Hooker, ${ }^{5}$ J. Holloway, ${ }^{8}$ J. Jiang, ${ }^{4}$ N. C. Lopes, ${ }^{3,4}$ H. Nakamura, ${ }^{3}$ P. A. Norreys, ${ }^{5}$ C. A. J. Palmer, ${ }^{1,2}$ P. P. Rajeev, ${ }^{5}$ \\ J. Schreiber, ${ }^{6,7}$ D. R. Symes, ${ }^{5}$ M. Wing, ${ }^{8}$ S. P. D. Mangles, ${ }^{3}$ and Z. Najmudin ${ }^{3, *}$ \\ ${ }^{1}$ The Cockcroft Institute, Keckwick Lane, Daresbury WA4 4AD, United Kingdom \\ ${ }^{2}$ Physics Department, Lancaster University, Lancaster LA1 4YB, United Kingdom \\ ${ }^{3}$ John Adams Institute for Accelerator Science, The Blackett Laboratory, Imperial College London, London SW7 2AZ, United Kingdom \\ ${ }^{4}$ GoLP/Instituto de Plasmas e Fusão Nuclear, Instituto Superior Técnico, Universidade de Lisboa, Lisboa 1049-001, Portugal \\ ${ }^{5}$ Central Laser Facility, Rutherford Appleton Laboratory, Chilton, Oxon OX11 OQX, United Kingdom \\ ${ }^{6}$ Fakultät für Physik, Ludwig-Maximilians-Universität München, Am Coulombwall 1, D-85748 Garching, Germany \\ ${ }^{7}$ Max-Planck-Institut für Quantenoptik, Hans-Kopfermann-Strasse 1, D-85748 Garching, Germany \\ ${ }^{8}$ High Energy Physics Group, University College London, London WCIE 6BT, United Kingdom
}

(Received 17 October 2017; published 19 June 2018)

\begin{abstract}
We report on the depletion and power amplification of the driving laser pulse in a strongly driven laser wakefield accelerator. Simultaneous measurement of the transmitted pulse energy and temporal shape indicate an increase in peak power from $187 \pm 11 \mathrm{TW}$ to a maximum of $318 \pm 12 \mathrm{TW}$ after $13 \mathrm{~mm}$ of propagation in a plasma density of $0.9 \times 10^{18} \mathrm{~cm}^{-3}$. The power amplification is correlated with the injection and acceleration of electrons in the nonlinear wakefield. This process is modeled by including a localized redshift and subsequent group delay dispersion at the laser pulse front.
\end{abstract}

DOI: $10.1103 /$ PhysRevLett.120.254801

Laser wakefield accelerators (LWFA) [1] can now produce electron beams with particle energies greater than $\mathrm{GeV}$ from centimeter scale interaction lengths [2-4]. In a LWFA, a high-intensity laser pulse propagating through a plasma initiates a plasma wave, which exhibits extremely high longitudinal electric fields. Numerous methods have been demonstrated to inject particles within a LWFA [5-8]. Of these, self-injection in the highly nonlinear "bubble" or "blowout" regime [2,9-11] is amongst the simplest and thus most common. By using self- [12] or external guiding [13], it is possible to maintain the LWFA far beyond the normal Rayleigh diffraction length. However, the eventual energy gain of electrons by the wakefield is limited either by dephasing or by depletion of the driving laser pulse [14].

Dephasing occurs when electrons outrun the wakefield, which is usually said to move at the linear group velocity of the laser pulse in the plasma, $v_{g}=c \sqrt{1-\omega_{p}^{2} / \omega_{0}^{2}}$, where $\omega_{p}$ and $\omega_{0}$ are the plasma and laser angular frequencies, respectively. For a linear relativistic plasma wave (i.e., with wavelength $\lambda_{p}=2 \pi c / \omega_{p}$ and Lorentz factor $\gamma_{\phi} \approx \sqrt{n_{c} / n_{e}} \gg 1$, where $n_{c}=\epsilon_{0} m_{e} \omega_{0}^{2} / e^{2}$ is the critical density), the dephasing length is $L_{d}=\gamma_{\phi}^{2} \lambda_{p}=\left(\omega_{0} / \omega_{p}\right)^{2} \lambda_{p}$.

For a short duration laser pulse driving a nonlinear wakefield (pulse length $\sigma_{t}<\lambda_{p} / c$ and normalized vector potential $a_{0} \gg 1$ ), plasma electrons are pushed outward by the front of the pulse such that the rear of the pulse propagates in an ion cavity. Pump depletion occurs at the front of the laser pulse as energy is coupled into the plasma wave or lost due to diffraction. As a result of this localized depletion, the laser rapidly evolves to have a sharp rising edge, which etches back through the pulse $[15,16]$. Decker et al. [15] showed that the velocity of this pulse front etching is $v_{\text {etch }}=c \omega_{p}^{2} / \omega_{0}^{2}$ in the group velocity frame of the laser. The depletion length is then

$$
L_{d p} \approx\left(\omega_{0}^{2} / \omega_{p}^{2}\right) \sigma_{t} c
$$

For a near-resonant pulse, $c \sigma_{t} \approx \lambda_{p}$; then, $L_{d p} \approx L_{d}$. So depletion should not limit electron energy gain. However, because of pulse front etching, the effective laser pulse velocity is reduced, such that the plasma wave phase velocity becomes $v_{\phi} / c=\left(v_{g}-v_{\text {etch }}\right) / c \approx 1-\frac{3}{2}\left(\omega_{p}^{2} / \omega_{0}^{2}\right)$ for $\omega_{p} \ll \omega_{0}$. This reduces the dephasing length to $L_{d}=$ $\frac{2}{3}\left(\omega_{0} / \omega_{p}\right)^{2} \lambda_{p}$, which limits the maximum electron energy gain [14].

Although guiding has been demonstrated up to $L_{d p}$ for a range of plasma densities [17], no quantitative measurement of pump depletion in nonlinear LWFA has been reported. In addition, the pulse front etching model does not include laser power amplification that is observed in numerical simulations [18]. Pulse shortening of LWFA drive pulses has been previously reported $[19,20]$ but not the power amplification, which is vital for self-injection of electrons [21-25].

In this Letter, we present measurements of energy depletion and pulse compression of a relativistic $\left(a_{0} \gg 1\right)$ 
short-pulse $\left(c \sigma_{t}<\lambda_{p}\right)$ laser in a self-guided LWFA. We also report the first direct measurements of power amplification of the driving laser pulse. These results are modeled by considering group velocity dispersion of the laser pulse as the leading edge is redshifted. The power amplification was found to be coincident with the onset of electron selfinjection, confirming its vital role in this process.

The experiment (see Supplementary Material for setup figure [26]) was performed using the Gemini laser [27], interacting with a supersonic helium gas jet, at electron densities of up to $n_{e}=4 \times 10^{18} \mathrm{~cm}^{-3}\left(0.007 n_{c}\right)$. Each linearly polarized laser pulse, of wavelength $\lambda_{0}=800 \mathrm{~nm}$, contained $11 \pm 1 \mathrm{~J}$ of energy in a duration of $t_{\mathrm{FWHM}}=$ $51 \pm 3$ fs. The laser was focused onto the front of the gas target with an $f / 20$ parabolic mirror at a peak $a_{0} \simeq 3.0$.

A magnetic electron spectrometer was used to measure the spectrum of the accelerated electron beam. The laser pulse at the exit plane of the plasma was imaged with a pair of $f / 10$ spherical mirrors at a resolution of $10 \mu \mathrm{m}$ over a field of view of $902 \mu \mathrm{m} \times 675 \mu \mathrm{m}$. The transmitted energy was measured by integrating the counts on the camera, which was cross calibrated with an energy diode. Also, a $5 \mathrm{~mm}$ diameter area near the center of the transmitted beam, $\approx 1 / 20^{\text {th }}$ of the full beam diameter, was directed to two Grenouille (Swamp Optics) SHG-FROGs (second harmonic generation frequency resolved optical gating) [28]. These devices produce spectrally dispersed autocorrelations, from which the complete temporal intensity and phase information of the pulse was retrieved using an iterative algorithm.

The spectral window of the FROG diagnostics were limited, such that the first could measure to a lower pulse length limit of $10 \mathrm{fs}$, for a time-bandwidth limited pulse, while the second was restricted to $20 \mathrm{fs}$. Additional glass was placed in the beam path of the second FROG to create a known spectral phase offset between the two diagnostics. Only retrieved pulses with the correct time direction have the correct phase offset, and so the inherent time-direction ambiguity could be resolved. This process was possible for $n_{e}<0.6 \times 10^{18} \mathrm{~cm}^{-3}$, while at higher densities the spectrum became too broad for the second FROG. For these measurements, gradual changes of the pulse shape and Wigner [29] transforms with increasing density were used to determine the direction of time. The phase retrieval algorithm was performed 10 times, each time with a different random seed, and variations in the retrieved pulses were included in the measurement error. Shots with visibly poor retrievals, large FROG errors (rms relative pixel error $>0.02$ ), or unresolved time direction uncertainties are not included in the results. Out of 59 shots, 43 are included in the graph.

Example FROG traces and retrieved pulses are shown in Fig. 1. The pulses were observed to frequency downshift and temporally compress for increasing plasma density up to $n_{e}=1.5 \times 10^{18} \mathrm{~cm}^{-3}$ for a $15 \mathrm{~mm}$ diameter nozzle. Beyond this density, the pulse length increased again due to energy depletion of the laser pulse.

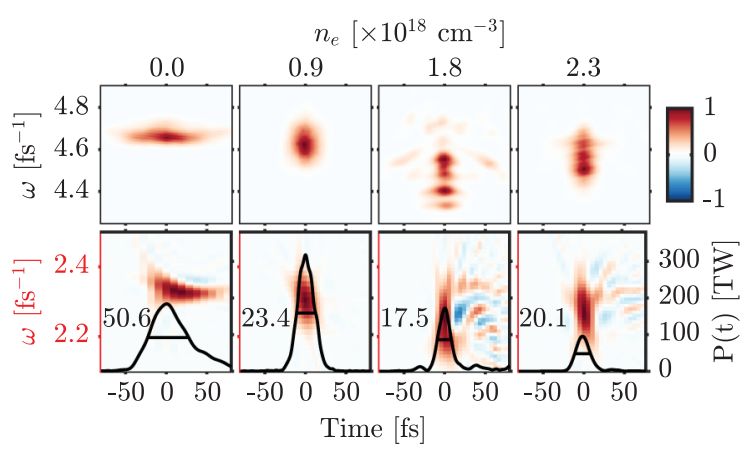

FIG. 1. (Top) FROG traces and (bottom) Wigner transforms with temporal profiles (black lines) for (left to right) $n_{e}=$ $(0,0.9,1.8,2.3) \times 10^{18} \mathrm{~cm}^{-3}$ and a nozzle diameter of $15 \mathrm{~mm}$. The Wigner transforms and temporal profiles have been corrected for diagnostic dispersion, representing the pulse at the plasma exit. The fs FWHM pulse durations are displayed by each pulse.

The results are shown as a function of plasma density for a $15 \mathrm{~mm}$ nozzle diameter in Fig. 2. For the plots of pulse energy transmission, transmitted pulse length, and peak power, each data point represents one measurement.

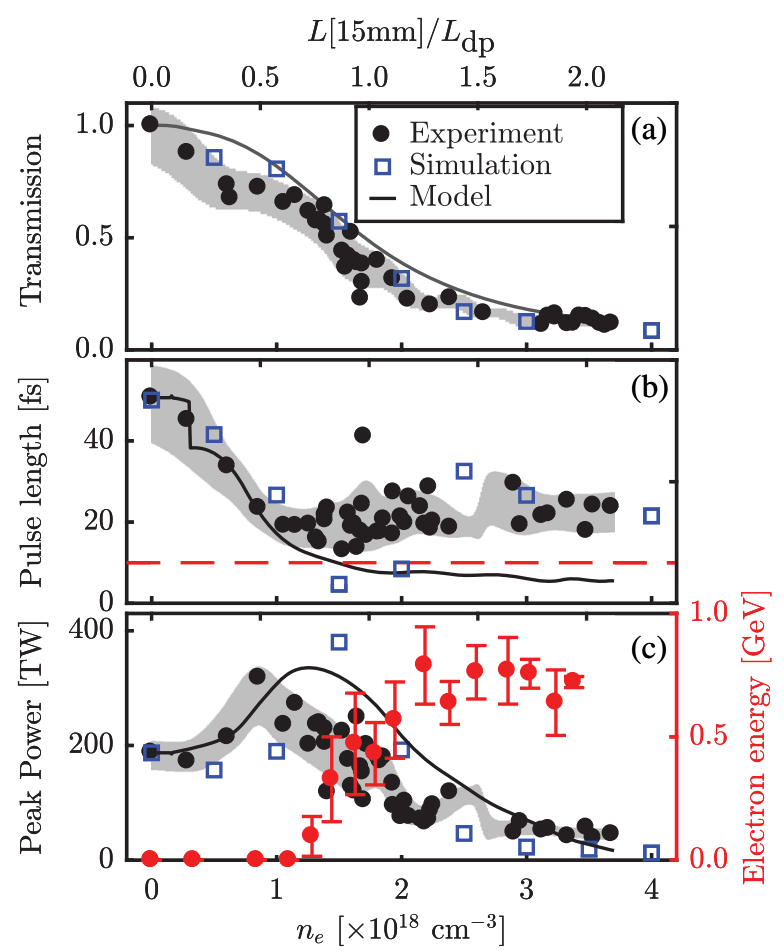

FIG. 2. Experimental and simulated (a) transmitted laser energy fraction, (b) pulse duration (FWHM), and (c) peak pulse power and maximum observed electron beam energy (red circles) versus plasma density for a $15 \mathrm{~mm}$ nozzle diameter. The gray shaded regions indicate rms error (statistical and measurement errors) of a moving average of the data points. The red dashed line in (b) is the instrument limit of the FROG for time-bandwidth limited pulses. The solid black lines are calculated from our pulse evolution model. 
The maximum electron energy [Fig. 2(c)] was taken as the highest point where the electron signal was above three times the rms background variation, after subtraction of the on-shot background. These values are averaged over multiple ( 4) shots within a density bin width of $0.2 \times 10^{18} \mathrm{~cm}^{-3}$, with error bars combining statistical and measurement errors. The ratio of the interaction length to $L_{d p}$ [Eq. (1)] is shown for comparison at the top of Fig. 2(a), with $\sigma_{t}=t_{\mathrm{FWHM}} / \sqrt{2 \ln (2)}$.

Transmitted laser energy decreased with increasing density [Fig. 2(a)]. About $50 \%$ of the energy was transmitted for $L=L_{d p}$, while the beam was only fully depleted for $L \simeq 2 L_{d p}$. Imaging of the exit plane showed a guided spot for $n_{e}>1 \times 10^{18} \mathrm{~cm}^{-3}$, while for $n_{e}>3 \times 10^{18} \mathrm{~cm}^{-3}$ only the unguided fraction of the laser energy was observed. The measured pulse duration [Fig. 2(b)] decreased from an initial $51 \pm 3$ fs to $\sim 20$ fs for $n_{e}>10^{18}$. For these higher densities, the spectral broadening resulted in spectral clipping in the FROG diagnostic, and so the retrieved pulse was longer than the input pulse. The shortest observed pulse length measurement in a single shot was $13.0 \pm 1.3$ fs for $n_{e}=$ $1.5 \times 10^{18} \mathrm{~cm}^{-3}$. The peak power of the laser pulse after the interaction [Fig. 2(c)] was calculated by setting the energy of the transmitted pulse equal to the time integral of the temporal pulse shape. The power was observed to increase, with a maximum at $n_{e}=0.9 \times 10^{18} \mathrm{~cm}^{-3}$, increasing from $187 \pm 11$ to $318 \pm 12$ TW. Wide-angle electron emission was produced for $n_{e}>0.2 \times 10^{18} \mathrm{~cm}^{-3}$, but the charge increased significantly for $n_{e}>1.1 \times 10^{18} \mathrm{~cm}^{-3}$ [Fig. 2(c)], where the maximum power enhancement was observed at the plasma exit. The maximum beam energies of $0.79 \pm 0.12 \mathrm{GeV}$, occurred at $n_{e} \simeq 2.3 \times 10^{18} \mathrm{~cm}^{-3}$, in line with scaling predictions [14].

Figure 3 shows laser measurements from three different nozzle diameters (temporal diagnostic was only available for the $15 \mathrm{~mm}$ nozzle). The similarity of the results when scaling the propagation length by the depletion length

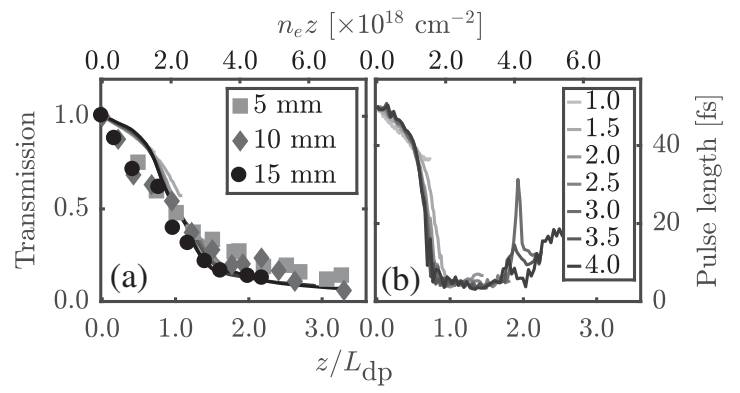

FIG. 3. Laser depletion (a) and pulse compression (b) as functions of the areal density (top axis) and the interaction length normalized by the depletion length (bottom axis). Experimental measurements (markers) are averaged over a $0.25 \times$ $10^{18} \mathrm{~cm}^{-2}$ bin width for three different nozzle diameters [inset in (a)]. Simulated values (lines) are given for different electron densities [inset in (b) in units of $10^{18} \mathrm{~cm}^{-3}$ ].
[Eq. (1)] indicates that the laser evolution is a function of the areal density $z n_{e} \propto z / L_{d p}$, over the covered density range.

The experiment was simulated using the OSIRIS [30] particle-in-cell code in 2D3V geometry. The simulation window moved at $c$ along the laser propagation direction and had dimensions of $200 \mu \mathrm{m} \times 200 \mu \mathrm{m}$ divided into $8000 \times 800$ cells in the pulse propagation $(z)$ and the transverse $(x)$ directions, respectively. The pulse envelope was modeled using a polynomial approximation to a Gaussian with $\tau_{\mathrm{FWHM}}=50 \mathrm{fs}$, focused to a spot width $w_{\text {FWHM }}=25 \mu \mathrm{m}$ and a peak $a_{0}$ of 3.0. The plasma target was $15 \mathrm{~mm}$ in length, including linear density ramps over $500 \mu \mathrm{m}$ at the entrance and exit of the plasma, approximating the experimental density profile, with four electron macroparticles per cell and stationary ions.

The simulated pulse properties at the plasma exit are shown alongside the experimental data in Figs. 2 and 3. The energy depletion and pulse compression proceeds at similar rates as in the experiment, but the pulse compresses to a minimum of 4 fs (below the experimental measurement limit) at $n_{e}=1.5 \times 10^{18} \mathrm{~cm}^{-3}$. At higher densities, the pulse length increases again, once almost all the laser energy depletes, and the short compressed peak in the laser field vanishes.

Figure 4(a) shows the propagation dependence of the onaxis plasma density modulation in the reduced group velocity $\left(v_{\phi}=v_{g}-v_{\text {etch }}\right)$ reference frame for $n_{e}=4 \times 10^{18} \mathrm{~cm}^{-3}$. The laser peak $a_{0}$, plotted as a red line, first increases via pulse compression and then decreases due to pump depletion. Self-focusing is observed in the first $1 \mathrm{~mm}$ of plasma, after which a stable guided spot size is reached. The density peak coinciding with the leading edge of the laser

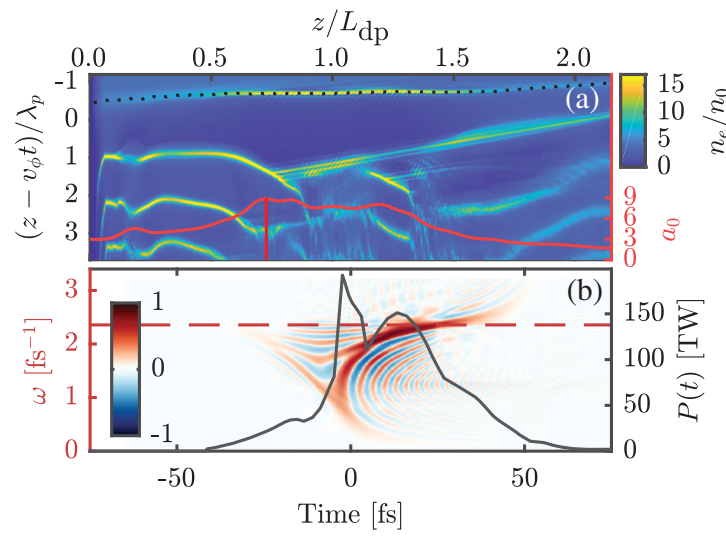

FIG. 4. (a) On-axis electron density map (image) in a frame moving at $v_{\phi}=v_{g}-v_{\text {etch }}$ and peak $a_{0}$ of the laser (red line), as functions of propagation distance in a simulation with $n_{e}=4 \times 10^{18} \mathrm{~cm}^{-3}$. The first maxima of the plasma wave is overlaid with a black dashed line. (b) Wigner transform (blue-red) of the laser pulse overlaid with the temporal intensity profile at $z=0.7 L_{d p}(3.9 \mathrm{~mm})$. The red horizontal line shows the initial laser frequency. 
pulse moves at close to the reduced group velocity, as represented by a horizontal line in the coordinate frame of the figure, in agreement with the pulse front etching model [15]. Deviation from this velocity is seen at early times, before the sharp front of the driving pulse forms, and at late times, once the laser is mostly depleted.

Rapid compression of the driving pulse occurs $4 \mathrm{~mm}$ into the target, increasing the plasma bubble radius since $r_{b} \propto$ $\sqrt{a_{0}}$ [31]. During this stage of the interaction, the effective phase velocity of the back of the wake decreases from $\gamma_{\phi}=$ 20 to $\gamma_{\phi}=7$. This coincides with self-injection of plasma electrons [32], as seen by the straight lines originating from the back of the first plasma wave and advancing relative to the plasma wave as the simulation progresses. The injection occurs over a short propagation distance, populating a narrow phase region of the wakefield, similar to injection mechanisms that use tailoring of the target density profile to modify the plasma wave phase velocity [21,33,34].

The pulse frequency shift and compression is illustrated by the Wigner transform of the simulated laser pulse at $z=4 \mathrm{~mm}$ in Fig. 4(b). The pulse is largely redshifted at the first density maximum of the plasma wave, close to the maximum of the laser intensity, as described by Schreiber et al. [20].

In the simple picture of pulse front etching, the leading edge of the laser pulse continually moves back through the pulse, locally reducing the laser power to zero. In reality, as photons at the leading edge are redshifted, they will begin to slip back through the pulse due to group velocity dispersion, as illustrated in Fig. 4(b). Photons are able to drift back away from the depletion region once their velocity is less than that of the laser pulse front. Equating the group velocity of a redshifted photon to the reduced group velocity of the laser pulse front, $v_{g}\left(\omega_{\min }\right)=v_{g}\left(\omega_{0}\right)-v_{\text {etch }}$, gives the minimum frequency reached by these photons as $\omega_{\min }=\omega_{0} / \sqrt{3}$. Once the power of the leading edge of the laser pulse drops below the critical power for self-focusing $P_{c}$, then it will diffract and no longer drive a plasma wave. Therefore, the energy coupled into the plasma from a local region in the power profile $W=(1-1 / \sqrt{3})\left[P(t)-P_{c}\right] \Delta t$. The remaining energy $(1 / \sqrt{3})\left[P(t)-P_{c}\right] \Delta t$ is transported back through the pulse by these redshifted photons into a region of low plasma density, where the group velocity dispersion is much smaller. This leads to an increase in power behind the depletion front and thereby modifies the energy depletion rate.

Numerical calculations were performed by stepping though the initial power profile $P(t)$ from the first point at which $P(t)>P_{c}$ for a given plasma density, reducing the power at this point to $P(t)=P_{c}$. The undepleted fraction of this energy $\left((1 / \sqrt{3})\left[P(t)-P_{c}\right] \Delta t\right)$ is added to the following region of the pulse, averaged over $\lambda_{p} / 4$ to approximate the effect of group velocity dispersion. The pulse energy, duration, and peak power after propagating $13 \mathrm{~mm}$ are calculated for each plasma density and are plotted as black lines in Fig. 2.

The numerical model predicts the energy depletion rate observed experimentally. The pulse compression is well reproduced until $n_{e}>1 \times 10^{18} \mathrm{~cm}^{-3}$, where the pulse length reaches a value lower than can be measured experimentally. The power amplification effect is also matched by the model, predicting a maximum power for $n_{e}=1.3 \times 10^{18} \mathrm{~cm}^{-3}$. Though, at this density, the experimental measurement is instrument limited because the pulse spectrum was broader than spectral range of the FROG diagnostic.

For a Gaussian laser pulse with the initial peak power $P_{0}>P_{c}$, maximum power amplification is reached when the pulse is etched to approximately the midway point, and the laser pulse energy is reduced by $\sim 50 \%$. This occurs after an evolution length,

$$
L_{\mathrm{evol}}=\sigma_{t} c\left(\frac{2}{3} \frac{\omega_{0}^{2}}{\omega_{p}^{2}}\right) \sqrt{\frac{1}{2} \ln \left(\frac{P_{0}}{P_{c}}\right)},
$$

For moderate values of $P_{0} / P_{c}\left(n_{e}>0.6 \times 10^{18} \mathrm{~cm}^{-3}\right.$ in Fig. 2), the evolution length is approximately equal to the usually quoted depletion length $L_{d p}$, and the pulse is only fully depleted at $L \approx 2 L_{\text {evol }}$. Taking injection to occur first at the position $z_{\text {inj }}=L_{\text {evol }}$, pump depletion of a $180 \mathrm{TW} 50 \mathrm{fs}$ pulse occurs before dephasing for $n_{e}<4.2 \times 10^{18} \mathrm{~cm}^{-3}$. At the density for which the maximum electron energy was observed in the experiment $n_{e}=2.3 \times 10^{18} \mathrm{~cm}^{-3}$, injection occurs at $z_{\mathrm{inj}}=8.6 \mathrm{~mm}$, giving an acceleration length of $4.4 \mathrm{~mm}$. However, the initial pulse shape in the experiment was non-Gaussian, having a rapid rising edge and an extended falling edge. As a result, $L_{\text {evol }}$ was shortened to $7.2 \mathrm{~mm}$, with the consequence that more laser energy remained in the pulse, allowing the acceleration length to be extended to $5.8 \mathrm{~mm}$. Using this value, and the experimentally measured electron energy, the average acceleration gradient over this acceleration length was $\approx 140 \mathrm{GeV} \mathrm{m}^{-1}$.

Hence, our pulse evolution model demonstrates that the laser pulse evolution is heavily influenced by the initial pulse shape. By using a pulse with an initially sharp rising edge, the laser pulse peak power increases rapidly, triggering injection before much of the laser energy is lost. A slow falling edge will then extend the depletion length, which could allow a large $a_{0}$ to be maintained over a longer distance. In this way, it may be possible to tailor the pulse shape to optimize the injection and acceleration processes, benefiting the many applications of these accelerators, such as the generation of large numbers of $\mathrm{x}$ rays [35], gamma rays [36], and positrons [37].

All data created during this research are openly available from Lancaster University data archive at [38].

We would like to acknowledge technical support from the CLF staff. This research was supported by 
STFC (ST/P002056/1, ST/J002062/1, ST/P000835/1) and EPSRC (EP/I014462/1). We thank the OSIRIS consortium (UCLA/IST) for the use of OSIRIS. M. Wing acknowledges the support of DESY, Hamburg, and the Alexander von Humboldt Stiftung.

z.najmudin@imperial.ac.uk

[1] T. Tajima and J. M. Dawson, Laser Electron Accelerator, Phys. Rev. Lett. 43, 267 (1979).

[2] W. P. Leemans, A. J. Gonsalves, H.-S. Mao, K. Nakamura, C. Benedetti, C. B. Schroeder, C. Tóth, J. Daniels, D. E. Mittelberger, S. S. Bulanov, J.-L. Vay, C. G. R. Geddes, and E. Esarey, Multi-GeV Electron Beams from CapillaryDischarge-Guided Subpetawatt Laser Pulses in the SelfTrapping Regime, Phys. Rev. Lett. 113, 245002 (2014).

[3] X. Wang, R. Zgadzaj, N. Fazel, Z. Li, S. A. Yi, X. Zhang, W. Henderson, Y.-Y. Chang, R. Korzekwa, H.-E. Tsai, C.-H. Pai, H. Quevedo, G. Dyer, E. Gaul, M. Martinez, A. C. Bernstein, T. Borger, M. Spinks, M. Donovan, V. Khudik, G. Shvets, T. Ditmire, and M. C. Downer, Quasimonoenergetic laser-plasma acceleration of electrons to 2 GeV, Nat. Commun. 4, 1988 (2013).

[4] H. T. Kim, K. H. Pae, H. J. Cha, I. J. Kim, T. J. Yu, J. H. Sung, S. K. Lee, T. M. Jeong, and J. Lee, Enhancement of Electron Energy to the Multi-GeV Regime by a Dual-Stage Laser-Wakefield Accelerator Pumped by Petawatt Laser Pulses, Phys. Rev. Lett. 111, 165002 (2013).

[5] E. Esarey, C. Schroeder, and W. Leemans, Physics of laserdriven plasma-based electron accelerators, Rev. Mod. Phys. 81, 1229 (2009).

[6] T. P. Rowlands-Rees, C. Kamperidis, S. Kneip, A. J. Gonsalves, S. P. D. Mangles, J. G. Gallacher, E. Brunetti, T. Ibbotson, C. D. Murphy, P. S. Foster, M. J. V. Streeter, F. Budde, P. A. Norreys, D. A. Jaroszynski, K. Krushelnick, Z. Najmudin, and S. M. Hooker, Laser-Driven Acceleration of Electrons in a Partially Ionized Plasma Channel, Phys. Rev. Lett. 100, 105005 (2008).

[7] A. Pak, K. A. Marsh, S. F. Martins, W. Lu, W. B. Mori, and C. Joshi, Injection and Trapping of Tunnel-Ionized Electrons into Laser-Produced Wakes, Phys. Rev. Lett. 104, 025003 (2010).

[8] C. McGuffey, A. G. R. Thomas, W. Schumaker, T. Matsuoka, V. Chvykov, F. J. Dollar, G. Kalintchenko, V. Yanovsky, A. Maksimchuk, K. Krushelnick, V. Y. Bychenkov, I. V. Glazyrin, and A. V. Karpeev, Ionization Induced Trapping in a Laser Wakefield Accelerator, Phys. Rev. Lett. 104, 025004 (2010).

[9] S. P. D. Mangles, C. D. Murphy, Z. Najmudin, A. G. R. Thomas, J. L. Collier, A. E. Dangor, E. J. Divall, P. S. Foster, J. G. Gallacher, C. J. Hooker, D. A. Jaroszynski, A. J. Langley, W. B. Mori, P. A. Norreys, F. S. Tsung, R. Viskup, B. R. Walton, and K. Krushelnick, Monoenergetic beams of relativistic electrons from intense laser-plasma interactions, Nature (London) 431, 535 (2004).

[10] C. G. R. Geddes, C. S. Toth, J. Van Tilborg, E. Esarey, C. B. Schroeder, D. Bruhwiler, C. Nieter, J. Cary, and W. P. Leemans, High-quality electron beams from a laser wakefield accelerator using plasma-channel guiding, Nature (London) 431, 538 (2004).

[11] J. Faure, Y. Glinec, A. Pukhov, S. Kiselev, S. Gordienko, E. Lefebvre, J.-P. Rousseau, F. Burgy, and V. Malka, A laserplasma accelerator producing monoenergetic electron beams, Nature (London) 431, 541 (2004).

[12] A. G. R. Thomas, Z. Najmudin, S. P. D. Mangles, C. D. Murphy, A. E. Dangor, C. Kamperidis, K. L. Lancaster, W. B. Mori, P. A. Norreys, W. Rozmus, and K. Krushelnick, Effect of Laser-Focusing Conditions on Propagation and Monoenergetic Electron Production in Laser-Wakefield Accelerators, Phys. Rev. Lett. 98, 095004 (2007).

[13] D. J. Spence and S. M. Hooker, Investigation of a hydrogen plasma waveguide, Phys. Rev. E 63, 015401 (2000).

[14] W. Lu, M. Tzoufras, C. Joshi, F. Tsung, W. Mori, J. Vieira, R. Fonseca, and L. Silva, Generating multi-GeV electron bunches using single stage laser wakefield acceleration in a 3D nonlinear regime, Phys. Rev. ST Accel. Beams 10, 061301 (2007).

[15] C. D. Decker, W. B. Mori, K.-C. Tzeng, and T. Katsouleas, The evolution of ultra-intense, short-pulse lasers in underdense plasmas, Phys. Plasmas 3, 2047 (1996).

[16] J. Vieira, F. Fiúza, L. O. Silva, M. Tzoufras, and W. B. Mori, Onset of self-steepening of intense laser pulses in plasmas, New J. Phys. 12, 045025 (2010).

[17] J. E. Ralph, K. A. Marsh, A. E. Pak, W. Lu, C. E. Clayton, F. Fang, W. B. Mori, and C. Joshi, Self-Guiding of Ultrashort, Relativistically Intense Laser Pulses through Underdense Plasmas in the Blowout Regime, Phys. Rev. Lett. 102, 175003 (2009).

[18] D. F. Gordon, B. Hafizi, R. F. Hubbard, J. R. Peñano, P. Sprangle, and A. Ting, Asymmetric Self-Phase Modulation and Compression of Short Laser Pulses in Plasma Channels, Phys. Rev. Lett. 90, 215001 (2003).

[19] J. Faure, Y. Glinec, J. J. Santos, F. Ewald, J.-P. Rousseau, S. Kiselev, A. Pukhov, T. Hosokai, and V. Malka, Observation of Laser-Pulse Shortening in Nonlinear Plasma Waves, Phys. Rev. Lett. 95, 205003 (2005).

[20] J. Schreiber, C. Bellei, S. P. D. Mangles, C. Kamperidis, S. Kneip, S. R. Nagel, C. A. J. Palmer, P. P. Rajeev, M. J. V. Streeter, and Z. Najmudin, Complete Temporal Characterization of Asymmetric Pulse Compression in a Laser Wakefield, Phys. Rev. Lett. 105, 235003 (2010).

[21] S. Kalmykov, S. A. Yi, V. Khudik, and G. Shvets, Electron Self-Injection and Trapping into an Evolving Plasma Bubble, Phys. Rev. Lett. 103, 135004 (2009).

[22] D. H. Froula, C. E. Clayton, T. Döppner, K. A. Marsh, C. P. J. Barty, L. Divol, R. A. Fonseca, S. H. Glenzer, C. Joshi, W. Lu, S. F. Martins, P. Michel, W. B. Mori, J. P. Palastro, B. B. Pollock, A. Pak, J. E. Ralph, J. S. Ross, C. W. Siders, L. O. Silva, and T. Wang, Measurements of the Critical Power for Self-Injection of Electrons in a Laser Wakefield Accelerator, Phys. Rev. Lett. 103, 215006 (2009).

[23] S. Kneip, S. R. Nagel, S. F. Martins, S. P. D. Mangles, C. Bellei, O. Chekhlov, R. J. Clarke, N. Delerue, E. J. Divall, G. Doucas, K. Ertel, F. Fiuza, R. Fonseca, P. Foster, S. J. Hawkes, C. J. Hooker, K. Krushelnick, W. B. Mori, C. A. J. Palmer et al., Near-GeV Acceleration of Electrons by a Nonlinear Plasma Wave Driven by a Self-Guided Laser Pulse, Phys. Rev. Lett. 103, 035002 (2009). 
[24] A. Sävert, S. P. D. Mangles, M. Schnell, E. Siminos, J. M. Cole, M. Leier, M. Reuter, M. B. Schwab, M. Möller, K. Poder, O. Jäckel, G. G. Paulus, C. Spielmann, S. Skupin, Z. Najmudin, and M.C. Kaluza, Direct Observation of the Injection Dynamics of a Laser Wakefield Accelerator Using Few-Femtosecond Shadowgraphy, Phys. Rev. Lett. 115, 055002 (2015).

[25] M. S. Bloom, M. J. V. Streeter, S. Kneip, R. A. Bendoyro, O. Cheklov, J. M. Cole, A. Doepp, C. J. Hooker, J. Holloway, J. Jiang, N. C. Lopes, H. Nakamura, P. A. Norreys, P. P. Rajeev, D. R. Symes, J. Schreiber, J. C. Wood, M. Wing, Z. Najmudin, and S. P. D. Mangles, Bright X-ray radiation from plasma bubbles in an evolving laser wakefield accelerator, arXiv:1710.05740.

[26] See Supplemental Material at http://link.aps.org/supplemental/ 10.1103/PhysRevLett.120.254801 for experimental setup figure.

[27] C. J. Hooker, J. L. Collier, O. Chekhlov, R. Clarke, E. Divall, K. Ertel, B. Fell, P. Foster, S. Hancock, A. Langley, D. Neely, J. Smith, and B. Wyborn, The Astra Gemini project-A dual-beam petawatt Ti:Sapphire laser system, J. Phys. IV (France) 133, 673 (2006).

[28] R. Trebino, Frequency-Resolved Optical Gating: The Measurement of Ultrashort Laser Pulses (Springer, Boston, MA, 2000).

[29] E. Wigner, On the Quantum Correction For Thermodynamic Equilibrium, Phys. Rev. 40, 749 (1932).

[30] R. A. Fonseca, L. O. Silva, F. S. Tsung, V. K. Decyk, W. Lu, C. Ren, W. B. Mori, S. Deng, S. Lee, T. Katsouleas, and J. C. Adam, in Computational Science ICCS 2002 SE-36, Lecture Notes in Computer Science, Vol. 2331, edited by P. Sloot, A. Hoekstra, C. Tan, and J. Dongarra (Springer, Berlin, 2002), pp. 342-351.

[31] W. Lu, C. Huang, M. Zhou, W. B. Mori, and T. Katsouleas, Nonlinear Theory for Relativistic Plasma Wakefields in the Blowout Regime, Phys. Rev. Lett. 96, 165002 (2006).
[32] C. B. Schroeder, C. Benedetti, E. Esarey, and W. P. Leemans, Nonlinear Pulse Propagation and Phase Velocity of Laser-Driven Plasma Waves, Phys. Rev. Lett. 106, 135002 (2011).

[33] S. Bulanov, N. Naumova, F. Pegoraro, and J. Sakai, Particle injection into the wave acceleration phase due to nonlinear wake wave breaking, Phys. Rev. E 58, R5257 (1998).

[34] C. G. R. Geddes, K. Nakamura, G. R. Plateau, C. Toth, E. Cormier-Michel, E. Esarey, C. B. Schroeder, J. R. Cary, and W. P. Leemans, Plasma-Density-Gradient Injection of Low Absolute-Momentum-Spread Electron Bunches, Phys. Rev. Lett. 100, 215004 (2008).

[35] S. Kneip, C. McGuffey, J. L. Martins, S. F. Martins, C. Bellei, V. Chvykov, F. Dollar, R. Fonseca, C. Huntington, G. Kalintchenko, A. Maksimchuk, S.P. D. Mangles, T. Matsuoka, S. R. Nagel, C. A. J. Palmer, J. Schreiber, K. T. Phuoc, A. G. R. Thomas, V. Yanovsky, L. O. Silva et al., Bright spatially coherent synchrotron X-rays from a tabletop source, Nat. Phys. 7, 737 (2011).

[36] G. Sarri, D. J. Corvan, W. Schumaker, J. M. Cole, A. Di Piazza, H. Ahmed, C. Harvey, C. H. Keitel, K. Krushelnick, S. P. D. Mangles, Z. Najmudin, D. Symes, A. G. R. Thomas, M. Yeung, Z. Zhao, and M. Zepf, Ultrahigh Brilliance Multi-MeV $\gamma$-Ray Beams from Nonlinear Relativistic Thomson Scattering, Phys. Rev. Lett. 113, 224801 (2014).

[37] G. Sarri, K. Poder, J. M. Cole, W. Schumaker, A. Di Piazza, B. Reville, T. Dzelzainis, D. Doria, L. A. Gizzi, G. Grittani, S. Kar, C. H. Keitel, K. Krushelnick, S. Kuschel, S. P. D. Mangles, Z. Najmudin, N. Shukla, L. O. Silva, D. Symes, A. G. R. Thomas et al., Generation of neutral and highdensity electron-positron pair plasmas in the laboratory, Nat. Commun. 6, 6747 (2015).

[38] http://dx.doi.org/10.17635/lancaster/researchdata/216. 